\title{
Methodologies for Active Aging in the Manufacturing Sector
}

\author{
Rosanna Fornasiero ${ }^{1}$, Domenica Berdicchia ${ }^{2}$, Mario Zambelli ${ }^{3}$, \\ and Giovanni Masino ${ }^{2}$ \\ ${ }^{1}$ ITIA-CNR, Via Bassini 15, 20133 Milano, Italy \\ ${ }^{2}$ University of Ferrara, Via Savonarola, 9, 44100 Ferrara, Italy \\ ${ }^{3}$ Politecnico Calzaturiero s.c.a.r.l., Via Venezia, 62, 30100 Vigonza (Pd), Italy \\ rosanna.fornasiero@itia.cnr.it, \\ \{d.berdicchia,g.masino\}@unife.it
}

\begin{abstract}
The research project named "Flexibly Beyond" studied and experimented innovative models for the enhancement of the role of senior workers and prolongation of their working life. The research was based on the application of innovative methods and tools to the ageing society and in particular to the European manufacturing companies represented in the project by apparel and footwear sectors. The project was funded under the Innovative Measures of the art.6 of the European Social Fund (VS/2006/0353) and coordinated by Politecnico Calzaturiero. The real strength of the project was the large network including all the actors of the value chain which allows transferring the theoretical findings to practical level in SMEs manufacturing context.
\end{abstract}

\section{The Issue, the Actors, the Goals}

Population aging is one of the most relevant demographic trends in western societies. There are various factors that explain such process: the decrease in fertility, the advances in medical technology, the general improvement of the quality of life, and others. The size and scope of this phenomenon is such that a number of social and economic issues related to the aging of population can now be considered of strategic importance for all western countries ([1]; [2]). In each country, however, there are some peculiar issues that specifically characterize how such a problem is impacting the society and the economic environment. In Italy, for example, the future sustainability of the pension system is a very relevant issue, as well as the economic risk associated with the potential loss of crucial competencies held by senior workers in small and medium enterprises (SMEs), which represent the backbone of the Italian economy. Other countries face similar or slightly different problems, but the common theme is the rapidly increasing magnitude and scope of the aging issue. Recently, the European Commission started to promote activities and studies in order to find ways to turn this problem into an opportunity. In fact, while it is shared that senior citizens and workers do have a lot to offer for the competitiveness of economies and for the improvement of the quality of their own lives and the life of others as well, it is not so clear how to integrate effectively their needs and their skills and abilities within fastpaced, rapidly changing markets and societies. 
The literature on age management offers useful insights about a possible general approach to the problem, one that should coordinate and integrate macro-level institutional actions and policies with micro-level organizational and managerial practices (see for example [3] and [8]). However, concrete successful solutions are hard to observe and implement, since a number of elements seem to increase the complexity of designing actual practices. Conflicting goals, lack of managerial competences and resources, cultural obstacles, insufficient awareness of the urgency and the relevance of the problem, are among the main aspects that seem to create great difficulties when trying to translate a general approach into actual solutions. This is particularly true and relevant where the social and economic context is mostly constituted by (SMEs). Some specific actions have been proposed in other EU projects held under the art. 6 of ESF each with its peculiarities of implementation.

In this paper we describe the general methodology and approach developed within the "Flexibly Beyond" project, whose goal was to study and experiment innovative models for the enhancement of the role of senior workers and prolongation of their working life. A number of research activities and experimental actions have been carried out in order to help SMEs to change their approach to the age management problem, with a specific focus on the apparel and footwear industries. The project was funded under the Innovative Measures of the art. 6 of the European Social Fund (VS/2006/0353) and Politecnico Calzaturiero coordinated successfully most important stakeholders of the value chain for the definition and implementation of policies for the valorization of senior workers. Education and research institutions, universities, enterprises, industry associations, workers unions and public institutions were part of a multidisciplinary team managed by Politecnico Calzaturiero. The choice to include a variety of points of view and interests has to be considered an important strategy in order to have a holistic approach to the challenge of ageing working population.

Apparel and footwear sectors represent an ideal field for this kind of initiative, for at least two main reasons. First, in many European countries they are very relevant in economic and social terms, as they account for a large share of the overall manufacturing sector, both in terms of employment and all other major economic indicators. Second, in such industries there is a vast majority of SMEs whose competitiveness, both in the short and the long run, is highly dependent on their ability to preserve the core competences and skills mostly held by senior workers. It will be shown that this can be done applying innovative methods and tools integrating new technologies and organizational methods.

The project was carried out thanks to the collaboration of three EU countries (Italy, Germany and Great Britain), in order to propose a general model for designing and implementing those practices in a variety of entrepreneurial contexts. For that goal, the comparison of specific experiences and solutions throughout a number of different countries, cultures, industries and enterprises has played a crucial role.

\section{The "Flexibly Beyond" Model}

The fundamental background of the project is that no practical solution and experiment could succeed without a strong convergent commitment between all the stakeholders and all the relevant actors' interest. 
The first part of the project was based on general analysis of the contexts in which new organizational collaborative solutions and practices could be implemented and tested. In this stage, the points of view of various stakeholders have been considered and analyzed, with a particular focus on senior workers and company's managers and entrepreneurs.

The model is based on the deep desk analysis supported by on-field survey (with company managers, senior workers, public institutions) and aims to propose a system where traditional methods and innovative tools are combined together into an integrated approach to the problem [4].

During the project activities the Flexibly Beyond model was developed and validated by the partners. In fact, the model was proposed for validation to companies other than the project's partners. The model is thought to support companies, and in particular SMEs, willing to face the challenge of losing competences held by senior workers using low cost methods and tools. The model is based on two steps:

1. business process analysis based on guided questionnaires for managers and workers, with dedicated SWOT analysis, Risk Age Management Analysis supported by Senior Age Management, and schemes for dedicated focus groups;

2. experimentation toolbox including methods for improving working time arrangements, new technologies and management support devices, educational and training initiatives, innovative software and others.

The model was conceived on a multi dimensional approach since it includes tools for both senior workers and company management, both at strategic and operative level. This is very important because on the one hand, it shows that a great deal of attention was directed towards the cultural aspects to improve the managers' awareness about the relevance of human resources and, in particular, about how a proper management of senior workers' competences and skills represents a crucial element for the companies' present and future competitiveness. On the other hand, it shows the overall philosophy of the project based on the fact that general problem of age management in SMEs cannot be solved without a shared, strong commitment by all the parties involved. Many senior workers (blue collars) with an operative role in the company have a deep knowledge about the process and the tasks to be performed. This tacit knowledge is very important in sectors where quality of the product and the craftsmanship are crucial. Most of the time such knowledge is not formalized and it is shared with other workers through informal "training on the job" and "learning by doing" practices. But these practices are not enough anymore and need to be formalize with shared paths to create collaborative networks both internally to the company and across the companies.

The toolbox of the Flexibly Beyond project is based on three main areas of action:

- Education: The goal is to help management to define a company Human resources (HR) strategy based on the valorization of competences, to train senior workers to become trainers and educators for junior workers, and to help knowledge transfers within the enterprise. These actions are thought to enhance the importance of such matters both among managers and workers themselves. On one side workers need to understand the importance of their role in the company and at the same time they need to be motivated for the knowledge transfer process which has to be perceived not as a loss but as a 
gain of knowledge. On the other side SME managers need to acquire awareness on the importance of having a clear HR Management policy and in particular of a clear strategy for managing key senior workers holding key core competences.

- Knowledge formalization: The goal is to develop tools in order to support managerial awareness about the age management issues and the available tools to empower and valorize senior workers. Also, the implementation of tools to formalize and codify knowledge in order to facilitate its transmission from seniors to juniors.

- Systems for flexibility: The goal is to support companies with tools of organizational flexibility in order to facilitate the prolonging of seniors' working life, for example by allowing more autonomy in organizing their time between leisure and work.

In the table below the experimentation toolbox is shown by the dimensions of recipients and the proposed tools.

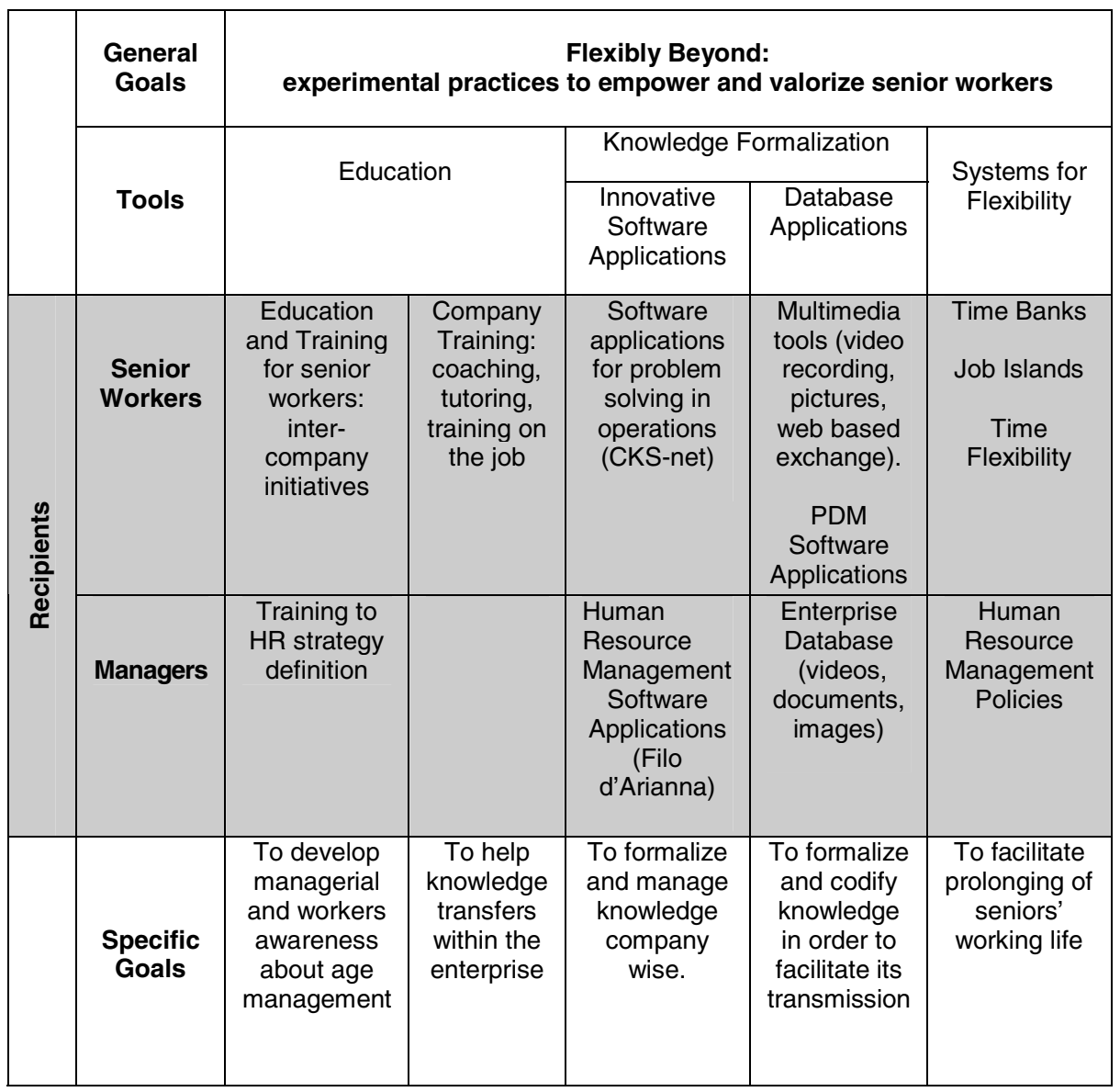

Fig. 1. Flexibly Beyond toolbox for experimentation 
In the next paragraphs, we will briefly describe some of the actions, and the related outcomes, that have been implemented through the "Flexibly Beyond" toolbox.

\section{Analysis of Organizational Structure}

In order to make sure that the innovative practices to implement and test were consistent with the specific needs of each company, a number of research and analysis have been carried out. Based on the collaborative network of the consortium, experts and consultants have been selected to help the enterprises' managers to evaluate their organizational and strategic situation, through in depth check-ups and SWOT analysis. In most cases, it was shown that enterprises involved in the project heavily depended on highly skilled senior workers, and that current human resource management practices were not sufficiently developed in order to deal with such a critical situation. In most SMEs, human resource management is considered as a mere administrative field, something that is not worth strategic considerations and investments. Thus, the project's initiative greatly helped the companies to develop their awareness about the limitations implied in such an approach to the general personnel policies, and to age management in particular. Also, the SAM risk analysis (Senior Age Management) helped to focus on specific risks that can identified and measured. Such a technique also allowed to profile each company in terms of what is currently done about the age management issue, what is not being done, and what are the possible consequences. From a comparative point of view, it can be shown that most enterprises, even the ones trying to consciously and carefully deal with the senior workers problem, face a relevant risk of losing crucial skills and competences with the retirement of some key senior workers; replacing or recreating those skills could be a very costly and difficult process.

Those costs and difficulties could be greatly reduced by some well planned-ahead actions. In some cases, it is not hard to imagine that the same practices that could provide to the companies means to prevent those problems and increase their competitiveness, they could also be considered by the senior workers as feasible ways to improve their status, their motivation and their satisfaction in the work place. In fact, several interviews conducted by the Project's staff to senior workers showed a widely shared positive attitude towards knowledge sharing and the extension of their work life, as long as arrangements are made in order to create adequate conditions and incentives, not only financial but also organizational ones. Thus, it seems that a certain convergence of goals and attitudes can be actually pursued and obtained. It is not an easy task, because many obstacles, especially cultural ones, on both sides, have to be overcome. It seems necessary to experiment with a wide variety of practices and changes, while at the same time taking into careful consideration the specific needs of each company.

\section{The Implementation of the Model}

The results of the first phase of the project have being used to categorize companies and to define specific needs and consequentially specific tools to be implemented. The "Flexibly Beyond" toolbox have been implemented in companies taking into 
consideration the specific requirements. Implementing the full toolbox in all the companies would have been out of the scope and not necessary. For this reason specific actions have been implemented. A relevant role in the project was played by the education and training initiatives, especially the "Competences Schools" that were created within some of the companies involved in the project. The goal was to establish and reinforce the role of senior workers as trainers and educators for the development of young workers' skills and abilities. While efforts of "training on the job" through unstructured means are commonly found, it has to be emphasized that a more structured approach is certainly necessary for different reasons:

- First, a formally structured approach to training process serves as way to promote throughout the company a culture of knowledge sharing, empowerment and responsibility for senior and junior workers.

- Second, it is also a way to reinforce and stress the relevance of a systematic, planned approach to human resource management.

- Third, a structured approach to education and training is better suited to create synergies with other kinds of tools and practices, like information technology tools and others that are described below.

A systematic approach to training and education, especially when senior workers take on a major role as trainers and educators, cannot, and should not, completely replace informal mechanisms of knowledge transmission. On the contrary, the "Competences Schools" should represent the ideal situation where informal knowledge transfers can be better managed and integrated with the organizational arrangement of the company and with its long term human resources strategy as well. The logic on which the "Competence School" approach is based acknowledges the impossibility of considering the training process just as "the transmission of explicit, abstract knowledge from the head of someone who knows to the head of someone who does not in surrounding that specifically exclude the complexities of practice" (see [5] p. 153). Instead, it affirms that "through experience of imitation and communication with other, [...] internal developmental potentialities are enacted and practiced until they are internalized as an independent development achievement" (see [6], p. 133).

Also, as a final point, the success of "Competences Schools" is strictly dependent on the ability and willingness of senior workers to act as effective trainers and educators. "Competences Schools" have been implemented through both company-specific and cross-company actions. An inter-company course has been implemented in order to motivate senior workers on the need to formalize their know-how. A "training for the trainers" action was implemented involving workers from different companies to create awareness that transfering know-how to other workers is not necessarily a losing strategy.

While it is crucial to improve the companies' ability to transfer and preserve the competences of senior workers, it is also important to provide effective tools for integrating such actions within a broader HRM strategy. The "Flexibly Beyond" model, for that goal, focused towards the development of innovative information technology tools for the management. One relevant example is "Filo d'Arianna", which is an IT tool designed to provide SMEs with a low cost platform for all major needs in terms of HR management. Such integration represents a particularly relevant feature, since it is necessary for companies not only to combine, from a strategic point of view, different 
aspects of HR management like routine tasks (such as payroll procedures), with non routine activities like career development and assessment, benefits and system for enhancing the role of senior workers. Thus, "Filo d'Arianna" represented a tool to allow managers to integrate HR management policies and activities into a platform that enhances the perception of strategic value of HR without compromising manageability.

Other relevant IT tools and practices have been implemented and tested in other companies. In the apparel sector companies, a tool of the PDM family (Product Data Management) has been customized and implemented in order to codify the procedures of important production activities, the "modeling" and the "prototyping" tasks, typical examples of areas where the experience of senior workers is absolutely crucial for the company. The use of PDM brings a number of advantages, e.g. the valorization of senior workers' know-how and the ability to create a database of well defined procedure that can be easily shared and communicated throughout the company.

Another example of innovative experiment has taken place in a small shoe company, where a Decision Support Application (designed according to the Case Based Reasoning technique) has been implemented in order to help workers to take effective decisions during some crucial stages of the production process, based on criteria of similarity with problems previously solved by senior workers [7]. Finally, a very interesting experience has been developed in other companies where the experience of senior workers was collected, stored and shared through multimedia techniques and procedures (video recording, interviews, etc.) as a simple and easy way to formalize and socialize tacit knowledge.

Generally speaking, technology played a relevant role in the experiments carried out within the "Flexibly Beyond" toolbox. This is not just because technology provides interesting opportunities per se, but also because it is absolutely crucial to disseminate the idea, especially among SMEs in traditional sectors like the clothing and shoe making industries, that technology is not just a way to innovate production processes, but also an effective way to better manage HR and to rethink creatively the organization to promote a cultural change in the overall managerial process of these enterprises. The implementation of such technological solutions was a way to reinforce collaborative networks within the company (increasing the interdependencies between different level workers).

It is worth noting that more traditional (but no less important) organizational changes have been implemented and tested in some of the companies involved in the project. The general goal was to improve the firms' organizational flexibility, so that senior workers could be facilitated and encouraged to extend their working life, to increase their commitment towards the sharing of knowledge and their new role as trainers for younger workers. A number of examples could be mentioned: the "time banks", which is mostly oriented towards the flexibility of working hours; the "work islands", which is an organizational arrangement for increasing of the autonomy of workers; "part-time" arrangements, "job sharing" practices, and other organizational solutions which should facilitate the integration between working time and private time.

\section{Conclusion}

It is becoming more and more obvious that western societies should carefully consider how to deal with some ongoing dramatic demographic changes, showing that 
senior citizens and workers are going to play an increasingly relevant role. Such a role does not have to be just a numeric burden on our society, instead it can be used as a positive resource, a new opportunity to improve not just the quality of life of senior citizens, but also to establish a closer knit, a more productive connection between generations, to tighten and strengthen the very fabric of our society as a whole. This might be not just the ethically right way to deal with the problem, but it also might be the only sensible, rational one. In fact, it is becoming clear that social and demographic issues are inextricably connected to economic problems. Many companies, in many different sectors and industries, are seriously threatened by the very real possibility of losing a great deal of their core competencies. The cost of recreating that knowledge might be very high, or even too high, for many of them.

The acceleration of changes (in all realms: social, technological, economic) is making more difficult, and more urgent, to take care of the difficult process of preserving traditional skills and competences and to passing them down to the new generations. In many economic sectors, innovation is not just a process of creating new knowledge, but also a combination of new and old knowledge. In other words, the aging problem in the socio-demographic field is also becoming a real, strategic issue, in the business arena.

The "Flexibly Beyond" project clearly showed that an effective answer to these enormous challenges which SMEs are facing requires a collective, multi-level and multi-disciplinary effort based on the implementation of tools with the support of a collaborative network based on internal relations (managers versus workers and workers themselves) and external relations with all the actors of the value chain (training providers, consultants, trade unions, etc). The support coming from these kind of actors is the basis for improving the performance of the company which is not only linked to productivity but also to quality of life, sustainability of the processes. And social sustainability is one important issue in a sector were human factor is still very strong. The evaluation of performance of the project is based on intangible factors and has indirect effects on production, but it has impact linked to the easen of information retrieving (shorter design process), easen of the knowledge transfer process (shorter learning time), number of workers still in the company after retiring age, etc.

Policy makers and institutions are responsible for adapting laws and regulations to allow industrial companies to face the challenge of active aging. Increasing the flexibility about the retirement timing and modalities probably would allow to design and implement organizational arrangements and innovations that would valorize the role of senior workers and citizens, improve the quality of their life, and facilitate the intergenerational transfer of knowledge, a crucial process for the economic development.

On the other side, the project itself helped companies to take into much higher consideration the problem of age management, and invest in policies, organizational innovation, and tools, specifically designed for that task. Even if one could argue that such a problem seems to concern particularly SMEs in traditional sectors, it is not hard to see that, instead, it should be seriously considered by all companies in every kind of industry. Also, it should taken into careful consideration by SMEs the need to integrate company-level to network-level actions. While the "Flexibly Beyond" project provided tools and methodologies at the company level, it also emphasized that, for various areas (e.g. training and education through Competence Schools) it is absolutely necessary a more collective approach, where collaborative networks between 
SMEs could represent the only efficient and effective approach, especially in areas where a critical mass of investments is required and where a district-like economy is present.

Finally, a variety of actions should be taken in order to develop and spread a new culture, a new way of thinking, a new public awareness, both inside the enterprises and in the society as a whole, about how senior citizens and workers should not represent and be considered as a problem, but instead as an opportunity, a positive resource, a chance to make structural changes that can actually benefit all sectors and all actors of the economy and the society. It could be the chance to imagine and implement innovations where the ethical and the rational thing to do will coincide.

Acknowledgments. The authors of this paper would like to acknowledge the project Flexibly Beyond (Flexibly Beyond: tools and methods to manage prolungation of the working life official n.VS/2006/0353), funded by the European Commission under the Innovative Measures of the art.6 of the European Social Fund. We would like to thank in particular Politecnico Calzaturiero, coordinator of the project, all project partners and consultants participating to the definition of the model and implementation of the actions during the year 2008 .

\section{References}

1. EU Commission: Aumentare il tasso d'occupazione dei lavoratori anziani e differire l'uscita dal mercato del lavoro, Comunicazione della Commissione al Consiglio, al Parlamento europeo, al Comitato economico e sociale europeo e al Comitato delle regioni, COM 146, Bruxelles (2004)

2. EU Commission (Comunicazione della): Il futuro demografico dell'Europa, trasformare una sfida in un'opportunità, COM 571, Bruxelles (2006)

3. Farinelli, D., Gubitta, P.: Il lavoro in età matura: come è fatto, come si può gestire, Franco Angeli, Milano (2007)

4. Berdicchia, D., Fornasiero, R., Masino, G., Zambelli, M.: Soluzioni organizzative per il prolungamento della vita lavorativa. Il lavoratore seniore nell'impresa e nella società. La Press, Venezia (2008)

5. Brown, J., Duguid, P.: Organizational Learning and Communities of Practice: Toward a unified view of working, learning, and innovation. In: Lesser, E.L., Fontaine, M.A., Jason, A. (eds.) Knowledge and Communities. Butterworth-Heinemann, Boston (1991)

6. Kolb, D.A.: Experiential learning: experience as the source of learning and development. Prentice-Hall, Inc., Englewood Cliffs (1984)

7. Bandini, S., Manzoni, S., Sartori, F.: Modelling Stories in the Knowledge Management Context to Improve Learning Within Organizations. In: Bramer, M. (ed.) Artificial Intelligence in Theory and Practice II. Springer, Heidelberg (2008)

8. Masino, G.: Le imprese oltre il fordismo. Retorica, illusioni, realtà, Carocci, Roma (2005) 